\title{
Desplazamiento altitudinal de las zonas productoras de cacao en el departamento Norte de Santander por efectos de las variaciones climáticas.
}

\section{Altitudinal displacement of cocoa-producing areas in the department of Norte de Santander due to the effects of climatic variations.}

\author{
José de Jesús Núñez-Rodríguez ${ }^{1}$, Omaira Mendoza-Ferreira² ${ }^{2}$ May Stefanny González-Verjel ${ }^{3}$, \\ Julio César Carvajal-Rodríguez ${ }^{4}$, Darcy Margarita Carrero-Carmona ${ }^{5}$ \\ 1,2,3,4 Universidad de Santander, Cúcuta - Colombia, \\ ${ }^{5}$ Universidad Nacional Experimental del Táchira, San Cristóbal - Venezuela \\ ORCID: ${ }^{1} \frac{0000-0002-4120-0215}{{ }^{2}} \frac{0000-0002-6535-6062}{{ }^{5}, \underline{0000-0002-6644-1488}}, \underline{0000-0002-9305-0212}, \underline{{ }^{4} 0000-0003-4517-8817}$,
}

Recibido: 22 de septiembre de 2020.

Aprobado: 11 de noviembre de 2020.

\begin{abstract}
Resumen - El objetivo de la investigación fue evaluar los efectos de las variaciones climáticas en las características físicas de las almendras de cacao (Theobroma cacao L.) en 6 gradientes altitudinales del departamento Norte de Santander, Colombia. Se seleccionaron 12 fincas, de pequeños productores, localizadas entre los $0 \mathrm{msnm}$ hasta los $1.200 \mathrm{msnm}$. Asimismo, se analizaron los registros de 36 años de temperaturas y precipitaciones medias obtenidos de una estación meteorológica del Instituto de Hidrología, Meteorología y Estudios Ambientales (IDEAM), ubicada a $50 \mathrm{msnm}$ dentro del área de influencia de las zonas cacaoteras evaluadas. En cada unidad de análisis se caracterizaron muestras de 300 gramos de almendras secas, de la cosecha de marzo-junio 2019, a las cuales se les determinó el peso de 100 almendras y el porcentaje de cascarilla. Los datos fueron analizados a través de estimadores descriptivos, ANAVAR y la Prueba de Tukey. Los resultados evidenciaron incrementos significativos en las temperaturas de $0.0315^{\circ} \mathrm{C} /$ año y variaciones en el peso de las almendras significativas, $\mathrm{p}<0.01, \mathrm{Fc}=33.4$, infiriéndose la aparición de 2 franjas climáticas para la producción de cacao en el departamento, la primera ubicada entre los 0 a los 600 msnm con granos medianos y grandes y altos contenidos de cascarilla y; la segunda, situada sobre los 600 hasta los 1.200 msnm, con granos de tamaño grande y bajo porcentaje de cascarilla. En conclusión, se establece que las variaciones climáticas están generando efectos negativos en los principales municipios productores de cacao del departamento y, al mismo tiempo, abre nuevos espacios para el desarrollo del cultivo en los municipios con altitudes superiores a los $600 \mathrm{msnm}$.
\end{abstract}

Palabras Claves: Agricultura, Agroclimatología, altitud, cambio climático, producción, temperatura.

Abstract - The objective of the research was to evaluate the effects of climatic variations on the physical characteristics of cocoa beans (Theobroma cacao L.) at 6 altitudinal levels in the department of Norte de Santander, Colombia. Twelve smallholder farms were selected, located between 0 and 1,200 meters above sea level, and 36 years of average temperature and rainfall records were analyzed from a meteorological station of the Institute of Hydrology, Meteorology and Environmental Studies (IDEAM), located at 50 meters above sea level within the area of influence of the evaluated cocoa zones. In each unit of analysis, samples of 300 grams of dry almonds from the March-June 2019 harvest were characterized and the weight of 100 almonds and the percentage of shells were determined. The data were analyzed through descriptive estimators, ANAVAR and the Tukey Test. The results showed significant increases in temperatures of $0.0315^{\circ} \mathrm{C} /$ year and significant variations in the weight of the almonds, $\mathrm{p}<0.01, \mathrm{Fc}=33.4$, inferring the appearance of 2 climatic bands for the production of cocoa in the department, the first one located between 0 and 600 m.a.s.l. with medium and large beans and high contents of husk and; the second one, located above 600 to 1,200 m.a.s.l., with large beans and low percentage of husk. In conclusion, it is established that climatic variations are generating negative effects in the main cocoa-producing municipalities of the department and, at the same time, opening new spaces for the development of the crop in municipalities with altitudes higher than $600 \mathrm{~m}$.

Keywords: agriculture, agroclimatology, altitude, climate change, production, temperature. 


\section{INTRODUCCIÓN}

Estudios realizados por el Fondo Financiero de Proyectos de Desarrollo y el Instituto de Hidrología, Meteorología y Estudios Ambientales [1] indican que el sector agropecuario colombiano viene padeciendo graves afectaciones por causas de índole climático. La intensidad de los fenómenos de El Niño y La Niña ha sido muy fuerte, en los últimos 30 años, ocasionando pérdidas económicas importantes en el país. Uno de los sistemas productivos agrícolas que sufre los impactos negativos de las variaciones climáticas es el cacao (Theobroma cacao L.) al experimentar los efectos en su rendimiento [2] - [4], alteraciones en las variables fisiológicas y reproductivas de las plantas [5] - [6], migración e infestación de plagas, malezas y enfermedades hacia pisos altitudinales superiores [7] - [10] y disminución de los rendimientos y calidad de las cosechas [11] - [12].

Las variaciones extremas en las precipitaciones y en las temperaturas ocasionan estrés en las plantas [13], [14], [15], causando la muerte o merma en la producción por las inundaciones y/o sequias intensas y prolongadas. Estas presiones climáticas sobre el cultivo y los productores de cacao han comenzado a impulsar el desplazamiento de las áreas sembradas hacia gradientes altitudinales mayores en la búsqueda de mejores climas, suelos y agua ([16] que les garantice escenarios óptimos para el cultivo [17] y una producción rentable y sostenible. En Colombia, la región con mayor producción de cacao es la denominada montaña santandereana, que se ubica entre los pisos térmicos cálido y templado donde se cultiva cacao entre los $200 \mathrm{msnm}$ hasta $\operatorname{los} 1.200 \mathrm{msnm}$ ([18], en pequeñas a medianas unidades productivas (promedio de 3,3 hectáreas), bajos niveles de tecnología y enmarcado dentro de una economía de subsistencia [19], [20], [18]. Pór tanto, se plantea como objetivo de la evaluar los efectos de las variaciones climáticas en las características físicas (peso, \% de almendras puras y de cascaras) de las almendras de cacao (Theobroma cacao L.) cultivado bajo 6 gradientes altitudinales del departamento Norte de Santander.

\section{METODOLOGÍA O PROCEDIMIENTOS}

\section{a. Localización}

La investigación se realizó en los municipios El Zulia (16 msnm), San Cayetano (235 msnm), Cúcuta (320 msnm), Sardinata (320 msnm), Gramalote $(1.040 \mathrm{msnm})$ y Bucarasica $(1.552 \mathrm{msnm})$, pertenecientes al departamento de Norte de Santander, localizado en las coordenadas $06^{\circ} 56^{\prime} 42^{\prime}$ y $09^{\circ} 18^{\prime} 01^{\prime \prime}$ ' de latitud norte y los $72^{\circ} 01^{\prime} 13^{\prime \prime}$ y $73^{\circ} 38^{\prime} 25^{\prime}$ ' de longitud oeste, Colombia.

\section{b. Material vegetal y establecimiento del experimento}

Se utilizaron almendras secas de cacao provenientes de la cosecha marzo-junio de 2019, de la mezcla de los materiales vegetales CCN51, ICS91, FEAR5, IMS67, Tame, ICS60 e Híbridos, cultivadas y secadas por los agricultores en sus parcelas.

\section{c. Diseño experimental}

Se empleó un diseño completamente aleatorizado, siendo el factor tratamiento la división en 6 gradientes altitudinales representativos de las unidades productoras del departamento de Norte de Santander, quedando de la siguiente forma: 1) Inferiores a 200 msnm; 2) 201-400 msnm; 3) 401-600 msnm; 4) 601-800 msnm; 5) 801-1.000 msnm y; 6) 1.001-1.200 msnm.

\section{d. Descripción de tratamientos}

En cada gradiente altitudinal se seleccionaron 2 unidades experimentales (fincas) para un total de 12 fincas estudiadas. Se analizaron muestras de 300 gramos de almendras secas, con manejo postcosecha particular según su nivel tecnológico del agricultor, de las cuales se tomaron al azar y pesaron 100 almendras, en 3 repeticiones sin reposición. Asimismo, se seleccionaron al azar 20 almendras con el fin de generar una separación entre la cascarilla y la almendra para determinar los porcentajes de cascarillas (PC) y el peso de las almendras puras (PAP).

Se realizó un análisis de la información climatológica del período 1980-2015 obtenida de la Estación Meteorológica Tibú, identificada con el código 1603501, localizada en la latitud 8.638333 y -72.726667 de longitud (50 msnm), perteneciente al IDEAM y reportada en el estudio "Documentos de lineamientos técnicos para la gestión del cambio climático y un desarrollo bajo en carbono y resiliente al clima" [21], realizado por la Corporación Autónoma Regional de la Frontera Nororiental (CORPONOR) y la Universidad Francisco de Paula Santander (UFPS) en el año 2018, en 15 estaciones meteorológicas del departamento Norte de Santander.

Las variables climáticas analizadas fueron temperatura y precipitación media anual. La clasificación de los granos de cacao por gradiente altitudinal, de acuerdo al peso y porcentaje de cascarillas, se realizó con base a la metodología de la Federación Nacional de Cacaoteros [22].

\section{e. Análisis estadístico}

Los datos obtenidos fueron analizados estadísticamente en dos vías; la primera descriptiva mediante la utilización de estimadores de tendencia central y de dispersión y la segunda de tipo inferencial a través del uso del análisis de la varianza, ANAVAR, y en los casos con diferencias significativas de medias se desarrolló la Prueba de Comparaciones Múltiples de Tukey. Las tendencias de las temperaturas y precipitaciones medias fueron estimadas por CORPONOR y la UFPS mediante gráficos de dispersión con líneas de tendencia, utilizando un modelo de Regresión Simple, uniendo los valores de los 36 años de registros climatológicos. 


\section{RESULTADOS}

\section{a. Comportamiento climatológico de las áreas en estudio} [21].

En esta sección se analizan las tendencias de las temperaturas y precipitaciones medias, basados en los resultados del estudio realizado por

\section{Tendencias de las temperaturas medias anuales}

El comportamiento de las temperaturas medias durante 36 años de registros de la Estación Tibú, ubicada dentro de las principales zonas productoras de cacao del departamento se muestra en la Figura 1.

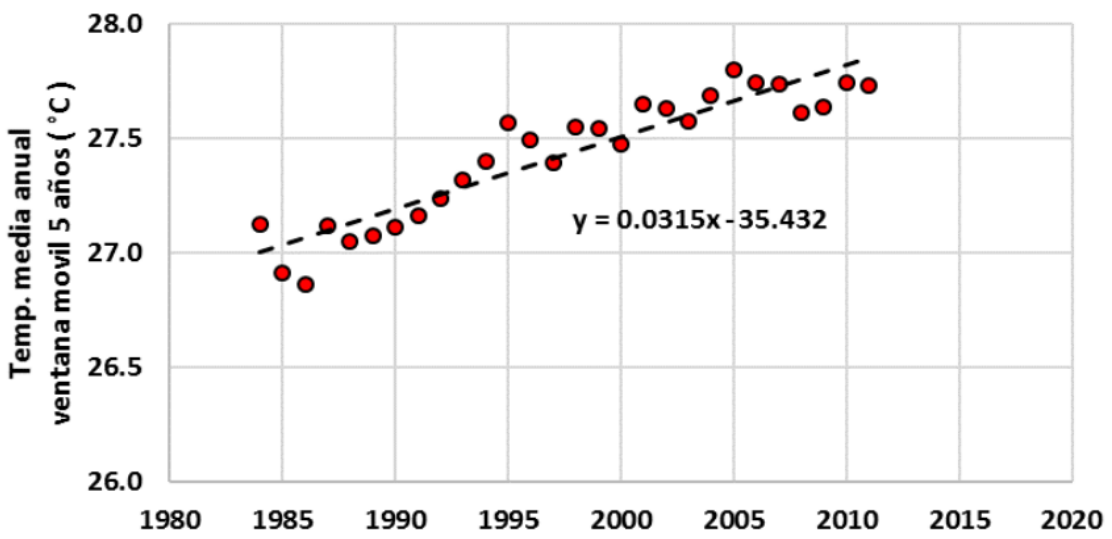

Figura 1: Tendencia de la temperatura media anual $\left({ }^{\circ} \mathrm{C}\right)$ registradas en la estación tibú (50 msnm). Fuente: [21].

La tendencia para la temperatura media muestra en la Figura 1 un incremento significativo, estimado de acuerdo al análisis de regresión en $\mathrm{y}=0.0315 \mathrm{x}-35.432$, es decir un aumento de $0.0315^{\circ} \mathrm{C} / \mathrm{año}$. En las tendencias de la temperatura al año 2040 se prevé variaciones entre +0.70 a $+0.79^{\circ} \mathrm{C}$ (Figura 2).

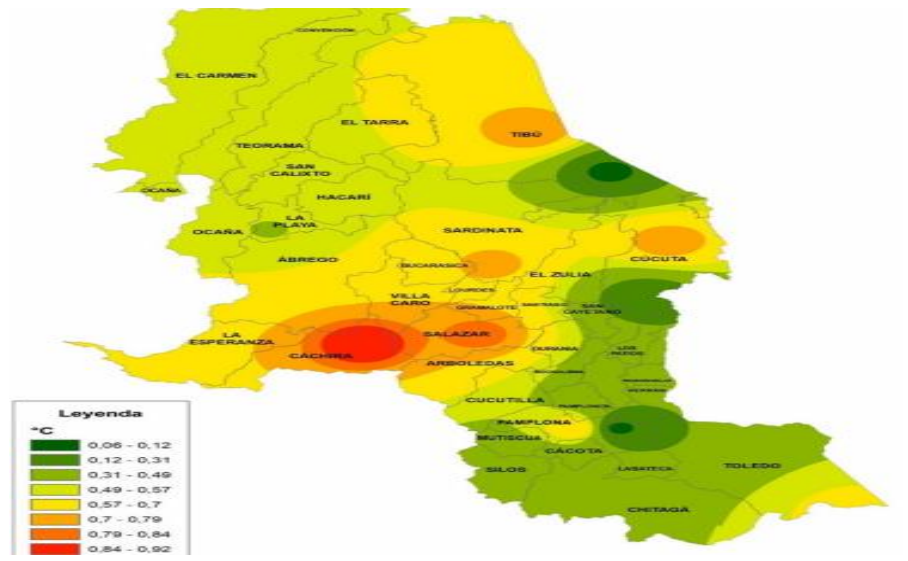

Figura 2: Estimación de las variaciones de la temperatura media del departamento Norte de Santander al año 2040. Fuente: [21].

\section{Tendencias de las precipitaciones medias anuales}

En la Figura 3, de acuerdo al estudio realizado por [21], se muestra la tendencia de las precipitaciones en la Estación Meteorológica de Tibú durante el período 1980-2015. 


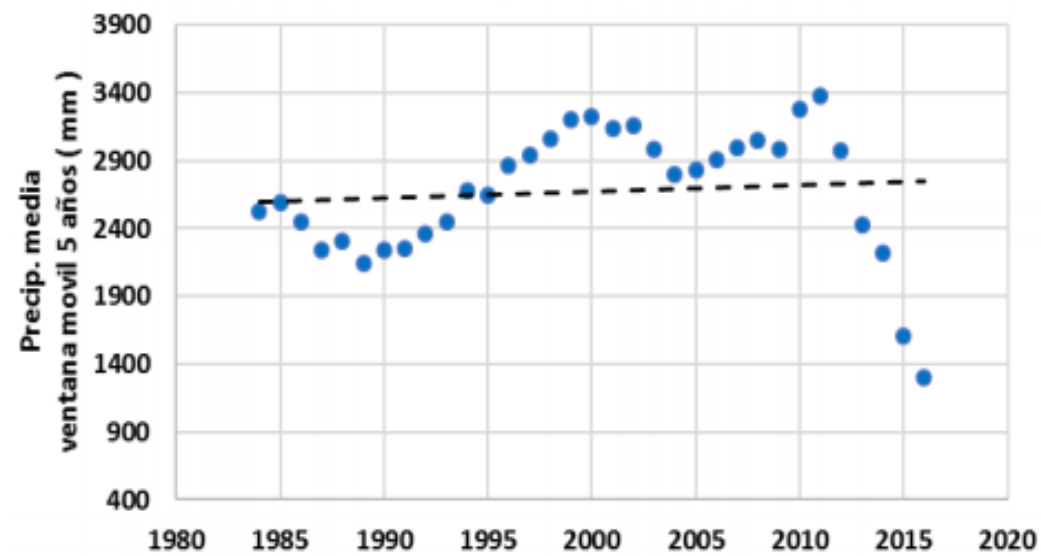

Figura 3: Tendencias de la precipitación media anual $\left({ }^{\circ} \mathrm{C}\right)$ registradas en la Estación Tibú $(50 \mathrm{msnm})$. Fuente: [21].

El análisis del comportamiento de las precipitaciones medias anuales no evidencia cambios significativos en los 36 años de registro. La recta de regresión lineal $\mathrm{y}=5.0203 \mathrm{x}-7368.7$, muestra un ligero incremento de las precipitaciones de la zona. Las variaciones de las precipitaciones para el año 2040 indican incrementos de las precipitaciones para la Estación Tibú entre +0.5 a +1.5 mm/año (Figura 4).

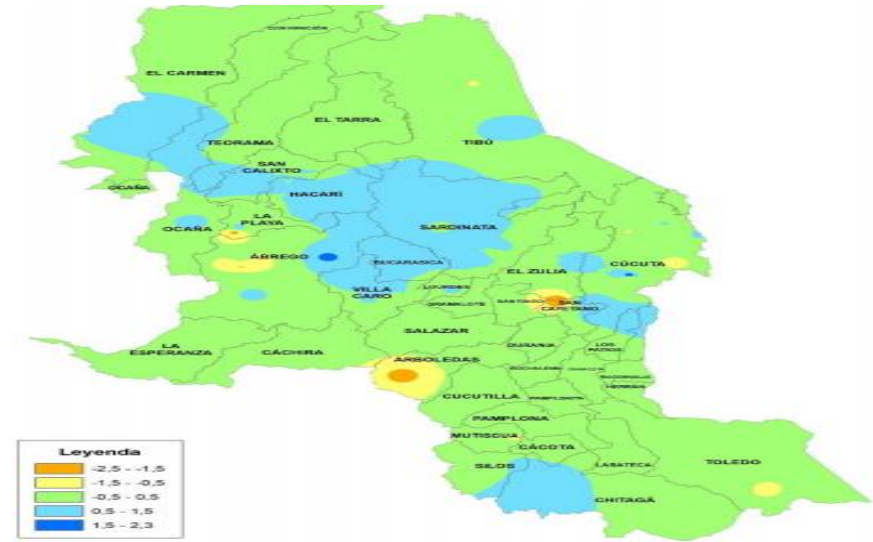

Figura 4: Estimación de las variaciones de las precipitaciones medias del departamento Norte de Santander al año 2040. Fuente: [21].

\section{b. Variaciones del peso de las almendras del cacao en los 6 gradientes altitudinales}

En este apartado se presentan los resultados de la evaluación del peso de 100 almendras por gradiente altitudinal y sus variaciones.

\section{Tendencias del peso de las almendras de cacao por gradiente altitudinal}

En la Tabla 1 y Figura 5 se observa que la media general del rendimiento de 100 almendras de cacao es de 134.28 g. con una desviación estándar de 12.05 g., lo que produce un coeficiente de variación del 8.97\%.

Tabla 1: Pesos de 100 almendras secas de cacao por gradiente altitudinal

\begin{tabular}{|c|c|c|c|c|c|c|c|c|}
\hline $\begin{array}{c}\text { Gradiente Altitudinal } \\
(\mathrm{msnm})\end{array}$ & $\begin{array}{c}\text { Media } \\
(\mathrm{g})\end{array}$ & $\begin{array}{c}\text { Desviación } \\
(\mathrm{g})\end{array}$ & $\begin{array}{c}\mathrm{CV} \\
(\%)\end{array}$ & $\begin{array}{c}\text { Min } \\
(\mathrm{g})\end{array}$ & $\begin{array}{c}\text { Max } \\
(\mathrm{g})\end{array}$ & $\begin{array}{c}\text { Intervalo de Confianza } \\
(95 \%)\end{array}$ & $\begin{array}{c}\text { Prueba Tukey } \\
\mathrm{P}<0.01\end{array}$ \\
\hline General & 134.28 & 12.05 & 8.97 & 109.00 & 157.00 & 130.20 & 138.35 & \\
\hline$<200$ & 115.17 & 6.27 & 5.45 & 109.00 & 123.00 & 108.58 & 121.75 & $\mathrm{~d}$ \\
\hline $201-400$ & 130.17 & 3.92 & 3.01 & 126.00 & 137.00 & 126.05 & 134.28 & $\mathrm{c}$ \\
\hline $401-600$ & 136.67 & 3.33 & 2.43 & 132.00 & 140.00 & 133.18 & 140.16 & $\mathrm{bc}$ \\
\hline $601-800$ & 130.17 & 3.43 & 2.64 & 126.00 & 135.00 & 126.57 & 133.77 & $\mathrm{c}$ \\
\hline $801-1.000$ & 145.83 & 8.09 & 5.54 & 136.00 & 157.00 & 137.35 & 154.32 & $\mathrm{ab}$ \\
\hline $1.001-1.200$ & 147.67 & 3.44 & 2.33 & 145.00 & 154.00 & 144.05 & 151.28 & $\mathrm{a}$ \\
\hline
\end{tabular}

Fuente: Elaboración propia. 


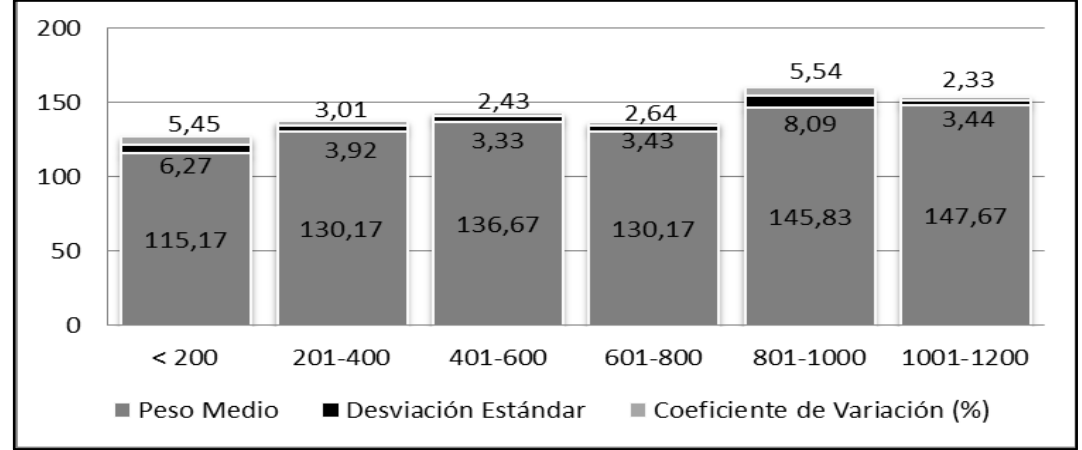

Figura 5: Pesos de 100 almendras secas de cacao por piso altitudinal.

Fuente: Elaboración propia.

Como se observa, el peso mínimo y máximo muestra un rango que va de 109.00 g. a 157.00 g. que en comparación con el intervalo de confianza del 95\%, se aleja de ambos límites de confianza. El análisis de la varianza determinó que existen diferencias significativas, $\mathrm{p}<0.01$, $\mathrm{Fc}=33.4$, entre los pesos medios de almendras para cada gradiente altitudinal. En al menos gradientes se detectaron diferencias reales en el rendimiento.

Los pesos de almendras de cacao cosechadas en los gradientes 5 y 6 resultaron los más altos, mientras que en el gradiente 1 se encuentran los pesos más bajos. Los gradientes 2, 3 y 4 producen almendras con pesos intermedios de $130.17 \mathrm{~g}$ a $136.67 \mathrm{~g}$. Este resultado corrobora que la tendencia observada en el análisis descriptivo obedece a diferencias reales entre los pesos medios de las almendras de cacao.

La prueba de Tukey determinó la existencia de 3 grupos homogéneos: el primero ubicado entre los gradientes desde los $401 \mathrm{msnm}$ a 600 msnm y de $801 \mathrm{msnm}$ a $1.200 \mathrm{msnm}$, con los mayores pesos; el segundo entre los gradientes de $201 \mathrm{msnm}$ a $800 \mathrm{msnm}$, pesos intermedios y; el tercero en gradientes menores a $200 \mathrm{msnm}$, con el menor promedio de peso de las almendras.

\section{Tendencias de los porcentajes de almendras puras y cascaras en las almendras de cacao en los gradientes altitudinales}

La determinación de los porcentajes de cascaras (PC) y almendras puras (PAP) en el peso de 20 almendras de cacao se muestran en la Tabla 2 y Figura 6.

Tabla 2: Porcentajes de almendras y de cáscaras en granos de cacao seco por piso altitudinal.

\begin{tabular}{|c|c|c|c|c|c|c|}
\hline Variable & $\begin{array}{c}\text { Gradiente } \\
\end{array}$ & Media (g) & Desviación & CV $(\%)$ & Min (g) & Max (g) \\
\hline \multirow{4}{*}{$\begin{array}{c}\text { Altitudinal (msnm) } \\
\text { Porcentaje de }\end{array}$} & General & 87.85 & 2.42 & 2.75 & 84.51 & 93.10 \\
\cline { 2 - 7 } & $<200$ & 85.77 & 1.78 & 2.08 & 84.51 & 87.03 \\
\cline { 2 - 7 } & $201-400$ & 85.72 & 0.34 & 0.40 & 85.48 & 85.96 \\
\cline { 2 - 7 } (PAP) & $401-600$ & 87.93 & 0.98 & 1.11 & 87.24 & 88.62 \\
\cline { 2 - 7 } & $601-800$ & 89.53 & 5.05 & 5.64 & 85.96 & 93.10 \\
\cline { 2 - 7 } & $801-1000$ & 88.59 & 1.00 & 1.13 & 87.88 & 89.29 \\
\cline { 2 - 7 } & General & 12.15 & 2.42 & 19.88 & 6.90 & 15.49 \\
\cline { 2 - 7 } & $<200$ & 14.23 & 1.78 & 12.52 & 12.97 & 15.49 \\
\cline { 2 - 7 } Porcentaje de & $201-400$ & 14.28 & 0.34 & 2.38 & 14.04 & 14.52 \\
\cline { 2 - 7 } Cáscaras (PC) & $401-600$ & 12.07 & 0.98 & 8.08 & 11.38 & 12.76 \\
\cline { 2 - 7 } & $601-800$ & 10.47 & 5.05 & 48.22 & 6.90 & 14.04 \\
\cline { 2 - 7 } & $801-1.000$ & 11.42 & 1.00 & 8.73 & 10.71 & 12.12 \\
\cline { 2 - 7 } & $1.001-1.200$ & 10.44 & 1.77 & 16.93 & 9.19 & 11.69 \\
\hline
\end{tabular}

Fuente: Elaboración propia.

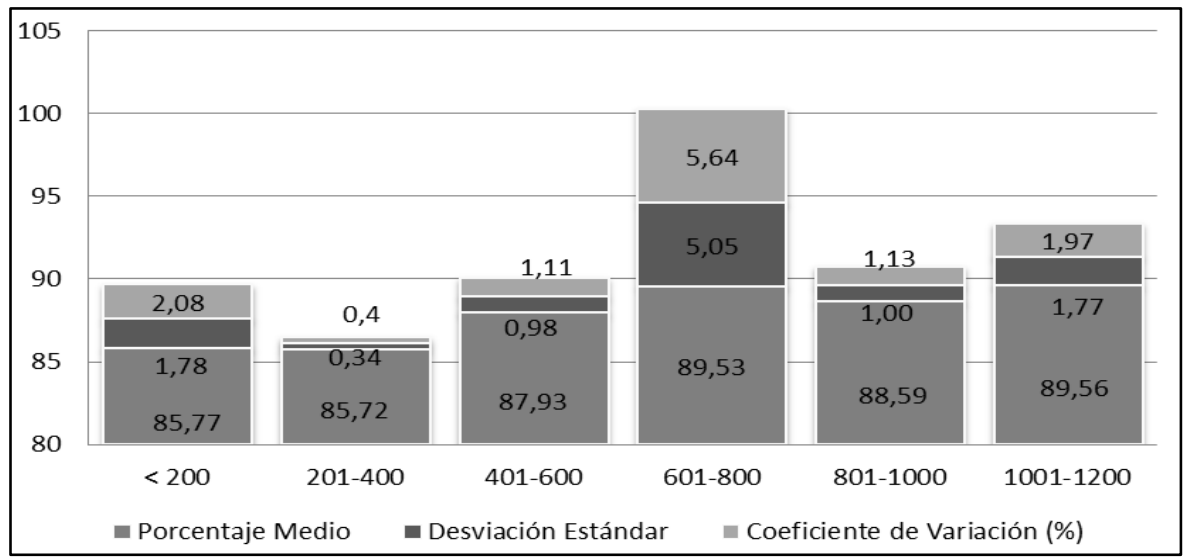

Figura 6: Porcentaje de almendras puras (PAP) por gradiente altitudinal (msnm).

Fuente: Elaboración propia. 

una ligera disminución de su valor; sin embargo, es notoria su tendencia al aumento gradual. Con respecto al porcentaje de las cáscaras (PC) la tendencia es a disminuir medida que aumenta la altitud.

En el análisis de la varianza no se detectó la existencia de diferencias significativas entre las medias de las variables en estudio entre los diversos gradientes altitudinales al arrojar un $\mathrm{Fc}=1.07$. Esto permite inferir que cualquiera sea el gradiente altitudinal, 0 a $1.200 \mathrm{msnm}$, las medias de las variables poseen el mismo comportamiento, la misma magnitud, de piso a piso. Las deferencias observadas entre las medias de los gradientes altitudinales pueden ser atribuibles al azar.

\section{c. Clasificación de la calidad de las almendras de cacao por gradiente altitudinal}

Dentro de la clasificación de FEDECACAO [22], los resultados obtenidos de la caracterización física de los granos de cacao en los 6 pisos gradientes en Norte de Santander se catalogan las calidades en dos: los granos que cumplen las condiciones para ser comercializados en los mercados nacionales (correspondientes a los granos con pesos promedios normales y altos y con alto contenido de cascarilla) y en el mercado internacional (almendras con pesos promedios altos y contenidos normales y bajos de cascarilla) (Tabla 3 ).

Tabla 3: Clasificación de los granos por piso altitudinal.

\begin{tabular}{|c|c|c|c|c|c|}
\hline $\begin{array}{l}\text { Gradiente altitudinal } \\
(\mathrm{msnm})\end{array}$ & $\begin{array}{l}\text { Peso medio } \\
\text { grano }(\mathrm{g})\end{array}$ & $\begin{array}{l}\text { Calificación } \\
\text { FEDECACAO }\end{array}$ & $\%$ Cascarilla & $\begin{array}{l}\text { Calificación } \\
\text { FEDECACAO }\end{array}$ & Descripción FEDECACAO \\
\hline$<200$ & 115.17 & Normal & 14.23 & Alto & $\begin{array}{l}\text { Granos medianos con alto contenido } \\
\text { de cascarilla, mercado nacional }\end{array}$ \\
\hline $201-400$ & 130.17 & Alto & 14.28 & Alto & $\begin{array}{l}\text { Granos grandes con alto contenido de } \\
\text { cascarilla, mercado nacional }\end{array}$ \\
\hline $401-600$ & 136.67 & Alto & 12.07 & Alto & $\begin{array}{c}\text { Granos grandes con alto } \\
\text { contenido de cascarilla, mercado nacional }\end{array}$ \\
\hline $601-800$ & 130.17 & Alto & 10.47 & Bajo & $\begin{array}{c}\text { Granos grandes con bajo contenido de cascarilla, } \\
\text { mercado internacional }\end{array}$ \\
\hline $801-1.000$ & 145.83 & Alto & 11.42 & Normal & $\begin{array}{c}\text { Granos grandes con mediano contenido de } \\
\text { cascarilla, mercado internacional }\end{array}$ \\
\hline $1.001-1.200$ & 147.67 & Alto & 10.44 & Bajo & $\begin{array}{c}\text { Granos grandes con bajo contenido de cascarilla, } \\
\text { mercado internacional }\end{array}$ \\
\hline
\end{tabular}

Fuente: Elaboración propia.

En la Tabla 3 se observa que las zonas productoras de cacao con altitudes menores a $600 \mathrm{msnm}$ la tendencia es a obtener almendras con Indice de Grano (IG) superiores a 1.2 (limite inferior permitido) pero los porcentajes de cascarillas tienden a ser mayores al $11 \%$ (limite inferior permitido) y; las zonas con altitudes superiores a los $600 \mathrm{msnm}$ los IG son altos y los porcentajes de cascarillas oscilan entre normal y bajo.

\section{IV.DISCUSIÓN DE LOS RESULTADOS}

Los resultados obtenidos de la caracterización física de las almendras de cacao en el departamento Norte de Santander son coherentes con otros hallazgos obtenidos en los países productores de cacao. El Banco Interamericano de Desarrollo [23], revela los impactos negativos y las tendencias en la distribución futura del cacao hacia nuevas áreas que le garanticen las condiciones ecológicas de supervivencia y sostenibilidad económica, al vislumbrar en el horizonte del año 2050 un cambio significativo en el paisaje geográfico del cultivo.

Asimismo, el Instituto Interamericano de Agricultura Tropical [24] expresa que, en el África, en los próximos 15 años, la competitividad se puede ver amenazada por el incremento en la temperatura como consecuencia del cambio climático, lo que podría disminuir la calidad y cantidad del cultivo de cacao. Con condiciones climáticas más cálidas, los árboles de cacao luchan por conseguir suficiente agua durante la época de crecimiento, restringiendo así el desarrollo de las vainas, que contienen el grano. Estudios, realizados en [25] develan en productores de cacao en Ghana (África), que los aumentos de temperatura y sequía afectan a la productividad del cacao.

Estas alarmas que coinciden con la realidad latinoamericana, donde se muestra que la producción de cacao debe ser atendida por el efecto de las variaciones climáticas en la calidad y producción [26], [27]. Asimismo, el Centro Agronómico Tropical de Investigación y Enseñanza (CATIE) [28], afirma que el cacao es muy sensible a los cambios en el clima y a los eventos asociados al cambio climático, como inundaciones, sequías, olas de calor, vientos intensos, lluvias y tormentas, que afectan al cacao, a sus patógenos, polinizadores y a otros organismos asociados. En Colombia, se probaron 9 especies de árboles de sombra, como sistema agro forestal, para garantizar la producción de cacao durante las épocas secas y húmedas al mantener la actividad fisiológica del cacao y constituir una buena práctica para la adaptación al cambio climático [29].

\section{CONCLUSIONES}

Las tendencias de 36 años de registros de la temperatura y precipitación media anual en la Estación Climatológica de Tibú, ubicada a 50 $\mathrm{msnm}$, dentro de las principales zonas productoras de cacao, reveló incrementos significativos de $+0.03315^{\circ} \mathrm{C} / \mathrm{año}$, y variaciones al año 2040 estimadas entre +0.70 a $+0.79{ }^{\circ} \mathrm{C}$. La tendencia en la precipitación media anual muestra un ligero incremento, no significativo, de las precipitaciones de la zona, y variaciones estimadas al 2040 entre +0.5 a $+1.5 \mathrm{~mm} / \mathrm{año}$. La temperatura para esta región actualmente es alta (alrededor de $27^{\circ} \mathrm{C}$ ) por lo que aumentos adicionales de temperatura constituyen una amenaza para la producción de cacao.

El peso promedio de 100 almendras secas por gradiente altitudinal, en el análisis de la varianza se determinó que existen diferencias significativas, $\mathrm{p}<0.01, \mathrm{Fc}=33.4$. Las almendras de cacao cosechadas en los gradientes 5 y 6 resultaron los más altos, mientras que en el 

gradiente 1 se encuentran los pesos más bajos. Los gradientes 2, 3 y 4 producen almendras con pesos intermedios de $130.17 \mathrm{~g}$ a $136.67 \mathrm{~g}$. Este resultado corrobora que la tendencia observada en el análisis descriptivo obedece a diferencias reales entre los pesos medios de las almendras de cacao.

Con respecto al porcentaje de almendras puras (PAP) y porcentaje de cáscaras por gradiente altitudinal no se observaron diferencias significativas.

Los resultados preliminares de esta investigación indican una tendencia a la segmentación de la franja productora de cacao del departamento Norte de Santander dos áreas: 1) Zona tradicional del cultivo de cacao (altitudes inferiores a los 600 msnm) con granos medianos y grandes y alto porcentaje de cascarilla, con aptitudes para el mercado nacional y; 2) Zona emergente para el cultivo de cacao (altitudes superiores a los $600 \mathrm{msnm}$ ) con almendras de tamaño grande y bajos contenidos de cascarilla, con calidades para el mercado internacional.

Se recomienda realizar estudios que incluyan mayor cantidad de muestras, diversidad de zonas biogeográficas, niveles tecnológicos de cultivo, diferentes variedades o tipos de cacao y tecnologías de manejo postcosecha para corroborar los resultados aquí presentados.

\section{VI.REFERENCIAS}

[1] FONADE- IDEAM, "Efectos del cambio climático en la producción y rendimiento de cultivos: evaluación del riesgo agroclimático por sectores", 2013. Disponible en: http://www.ideam.gov.co/documents/21021/21138/Efectos+del+Cambio+Climatico+en+la+agricultura.pdf/3b209fae-f078-4823-afa0$1679224 \mathrm{a} 5 \mathrm{e} 85$

[2] C. A. Harvey et al., "Climate change impacts and adaptation among smallholder farmers in Central America". Agriculture and Food Security, vol. 7, $\mathrm{n}^{\circ}$ 1, pp. 57, 2018, doi: https://doi.org/10.1186/s40066-018-0209-x

[3] K.R. Kgosikoma, P. Lekota \& O.E. Kgosikoma, "Agropastoralists' determinants of adaptation to climate change", International Journal of Climate Change Strategies and Management, vol. 10, n. 3, pp. 488-500, 2018, doi: https://doi.org/10.1108/IJCCSM-02-2017-0039

[4] R. Sada et al., "People's experience and facts of changing climate: impacts and responses", International Journal of Climate Change Strategies and Management, vol. 6, $\mathrm{n}^{\circ}$. 1, pp. 47-62, 2014, doi: https://doi.org/10.1108/IJCCSM-04-2013-0047

[5] S. M. Gourdji, A.M. Sibley, D.B. \& Lobell, D. B., "Global crop exposure to critical high temperatures in the reproductive period: historical trends and future projections", Environmental Research Letters, vol. 8, n . 2, pp. 1-10, 2013, doi: https://doi.org/10.1088/17489326/8/2/024041

[6] C. Nendel et al., (2019), "Modelling cropping systems under climate variability and change: impacts, risk and adaptation", Agricultural systems, vol. 159, pp. 139-143, 2019.

[7] L. H. Ziska et al.,"Climate change, carbon dioxide, and pest biology, managing the future: Coffee as a case study", Agronomy, vol.8, ${ }^{\circ}$ 8, pp. 152, 2018, doi: https://doi.org/10.3390/agronomy8080152

[8] L. A. Galindo et al., "Lepidópteros perforadores de los frutos del café", Apuntes Técnicos de Cenicafe, vol. 434, pp.1-8, 2013.

[9] M. Čačija et al., "Linking climate change and insect pest distribution: an example using Agriotes ustulatus Shall. (Coleoptera: Elateridae)", Agricultural and Forest Entomology, vol. 20, n 2, pp. 131-300, 2017, doi: https://doi.org/10.1111/afe.12259

[10] Y. Aguirre, "El desplazamiento de los pisos térmicos y el lenguaje semiótico de las plantas como una expresión de su estrés biológico: dos imaginarios sociales de la población caldense sobre los efectos generados por el cambio climático", Luna Azul, vol. 36, pp. 55-69, 2013.

[11] A. L. D'Agostino and W. Schlenker, "Recent weather fluctuations and agricultural yields: implications for climate change", Agricultural Economics, vol. 47, ${ }^{\circ}$ 1, pp. 159-172, 2016, doi: https://doi.org/10.1111/agec.12315

[12] M. A. Lachaud, B.E. Bravo-Ureta, y C.E. Ludena, "Agricultural productivity in Latin America and the Caribbean in the presence of unobserved heterogeneity and climatic effects", Climatic Change, vol. 143, pp. 445-460, 2017, doi: https://doi.org/10.1007/s10584-0172013-1

[13] T. Sun et al., "Stage-dependent temperature sensitivity function predicts seed-setting rates under short-term extreme heat stress in rice", Agricultural and Forest Meteorology, vol. 256-257, ${ }^{\circ}$ 5, pp. 196-206, 2018, doi: https://doi.org/10.1016/j.agrformet.2018.03.006

[14] L. Gu et al., "Impacts of precipitation variability on plant species and community water stress in a temperate deciduous forest in the central US", Agricultural and Forest Meteorology, vol. 217, n 15, pp. 120-136, 2016, https://doi.org/10.1016/j.agrformet.2015.11.014

[15] E. Cremonese et al., "Heat wave hinders green wave: The impact of climate extreme on the phenology of a mountain grassland", Agricultural and Forest Meteorology, vol. 247, pp. 320-330, 2017, doi: https://doi.org/10.1016/j.agrformet.2017.08.016

[16] S. Bakri, A. Setiawan and I. Nurhaida, "Coffee bean physical quality: The effect of climate change adaptation behavior of shifting up cultivation area to a higher elevation", Biodiversitas, vol. 19, n 2, pp. 413-420, 2018, doi: https://doi.org/10.13057/biodiv/d190208

[17] G. Gram et al., "Local tree knowledge can fast-track agroforestry recommendations for coffee smallholders along a climate gradient in Mount Elgon, Uganda”, Agroforestry Systems, vol. 92, n 6, pp. 1625-1638, 2018, doi: https://doi.org/10.1007/s10457-017-0111-8

[18] L. Quintana et al., "Caracterización de tres índices de cosecha de cacao de los clones CCN51, ICS60 e ICS 95, en la montaña santandereana, Colombia", Investigación Agraria y Ambiental, vol. 6, $\mathrm{n}^{\circ}$ 1, pp. 252-265, 2015, doi: https://doi.org/10.22490/21456453.1284

[19] N. Vásquez-Barajas et al., "Análisis económico del sector cacaotero en Norte de Santander, Colombia y a nivel internacional", Investigación, desarrollo e innovación, vol. 8, n 2, pp. 237-250, 2018, doi: https://doi.org/10.19053/20278306.v8.n2.2018.7963

[20] P. Galvis, "Aportes del sistema de calidad para la competitividad de la cadena productiva del cacao en el municipio del Carmen de Chucuri Santander, Colombia", Tesis de Especialización, Universidad Militar Nueva Granada. Colombia, 2018. Disponible en: https://repository.unimilitar.edu.co/bitstream/handle/10654/20082/GalvisPaolaAndrea2018.pdf?sequence=1\&isAllowed=y

[21] CORPONOR-UFPS, "Documentos de lineamientos técnicos para la gestión del cambio climático y un desarrollo bajo en carbono y resiliente al clima”, 2018. Disponible en: http://corponor.gov.co/corponor/PICCDNS/PRODUCTO_N_3_3206001.pdf

[22] FEDECACAO, "Guía técnica para el cultivo del cacao", Quinta edición, Bogotá, Colombia, 2012.

[23] Banco Interamericano de Desarrollo, "Agricultura y clima futuro en América Latina y el Caribe: Impactos sistémicos y posibles respuestas", 2014. Disponible en: https://publications.iadb.org/publications/spanish/document/Agricultura-y-clima-futuro-enAm\%C3\%A9rica-Latina-y-el-Caribe-impactos-sist\%C3\%A9micos-y-posibles-respuestas.pdf 
Desplazamiento altitudinal de las zonas productoras de cacao en el departamento Norte de Santander por efectos de las variaciones climáticas

[24] IICA, "Estado actual sobre la producción, el comercio y cultivo del cacao en América", 2016. Disponible en: file:///C:/Users/Usuario/Downloads/BVE17048806e.pdf

[25] L. Ameyaw et al., "Cocoa and Climate Change: Insights from Smallholder Cocoa Producers in Ghana Regarding Challenges in Implementing Climate Change Mitigation Strategies”, Forest, vol. 9, n 742, pp. 1-20, 2018, doi: http://dx.doi.org/10.3390/f9120742

[26] J. Albiño, "Influencia del cambio climático en la producción de los cultivos de cacao en el cantón Shushufindi", Tesis de Maestría, Universidad Andina Simón Bolívar, Ecuador, 2019. Disponible en: http://repositorio.uasb.edu.ec/handle/10644/6890

[27] C. Bunn et al., "Impacto del cambio climático en la producción de cacao para Centroamérica y El Caribe", CIAT, 2019, doi: https://doi.org/10.7910/DVN/QUKZTO

[28] W. Phillips, "Impacto del cambio climático sobre el cacao y sus enfermedades", VIII Foro Nacional de Cacao. Producción y Productividad: es nuestra responsabilidad .San Pedro Sula, Honduras, 2015. Disponible en: http://infocafes.com/portal/wpcontent/uploads/2016/10/impacto_del_cambio_climatico_en_el_cacao-wilbert-phillips.pdf

[29] G. Agudelo-Castañeda et al., "Desempeño fisiológico de nueve especies de cacao (Theoborma cacao L.) bajo la sombra de tres especies forestales en Santander, Colombia", Revista colombiana de ciencias hortícolas, vol. 12, n 1, pp. 223-232, 2018, doi: http://dx.doi.org/10.17584/rcch.2018v12i1.7341. 